\title{
Traduire
}

Ine autre perspective sur I t raduction

Revue française de la traduction

$240 \mid 2019$

Quand la politique s'en mêle

\section{Traduire en langue des signes française : un acte militant?}

\section{Aurélia Nana Gassa Gonga}

\section{(2) OpenEdition}

1 Journals

Édition électronique

URL : http://journals.openedition.org/traduire/1688

DOI : 10.4000/traduire.1688

ISSN : 2272-9992

Éditeur

Société française des traducteurs

\section{Édition imprimée}

Date de publication : 20 juin 2019

Pagination : 59-68

ISSN : 0395-773X

\section{Référence électronique}

Aurélia Nana Gassa Gonga, «Traduire en langue des signes française : un acte militant? », Traduire [En ligne], 240 | 2019, mis en ligne le 20 juin 2019, consulté le 06 février 2020. URL : http:// journals.openedition.org/traduire/1688; DOI : 10.4000/traduire.1688 


\section{Traduire en langue des signes française : un acte militant?}

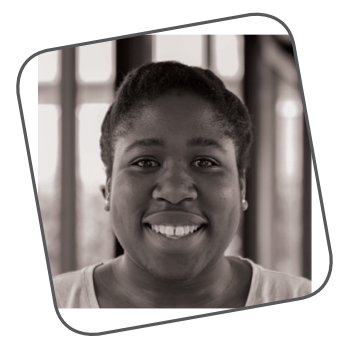

\section{Aurélia Nana Gassa Gonga}

Cet article se fait l'écho de celui publié par deux collègues interprètes en langue des signes française (LSF), en 2003: "Interpréter en langue des signes: un acte militant? (Quipourt et Gache 2003). Plus de quinze années plus tard, nous posons la même question du côté de la traduction: "Traduire en langue des signes française: un acte militant?» À l'heure où la frontière entre interprète et traducteur s'effrite peu à peu dans le champ de la traductologie des langues vocales, elle reste plus marquée dans celui de la traductologie des langues signées. Ainsi, les interprètes français (oral)/LSF sont rarement des traducteurs français (écrit)/LSF-vidéo, et vice-versa. L'usage et la formation veulent que les interprètes LSF soient entendants ${ }^{1}$, alors que les traducteurs LSF sont sourds. Ces derniers sont communément appelés traducteurs sourds. 
En France, on estime à environ 300000 le nombre de personnes sourdes ayant comme langue d'usage la LSF². Il est par ailleurs connu que les adultes sourds sont, très massivement, en grande difficulté dans l'écrit de la langue vocale ${ }^{3}$. Par conséquent, les sous-titres télévisés ne sont pas accessibles à la plupart d'entre eux. Dès lors, un accès à l'information passe nécessairement par une traduction vers la LSF, en l'occurrence LSF-vidéo dans le contexte audiovisuel que sont la télévision et l'internet.

Cependant, prenons l'exemple des attentats perpétrés le 13 novembre 2015 à Paris. La télévision, et indirectement le gouvernement français, n'a pas su tenir son rôle de pourvoyeur d'information à l'égard des citoyens français sourds: les flashs d'information spéciaux et l'allocution présidentielle n'ont pas été interprétés pour cette communauté. Dès le lendemain, la page Facebook «Fusillade à Paris en direct LSF${ }^{4}$ » a été créée à l'initiative de deux journalistes sourdes, Laurène Loctin et Pauline Stroesser (voir figure 1). Elles se sont entourées d'inter-

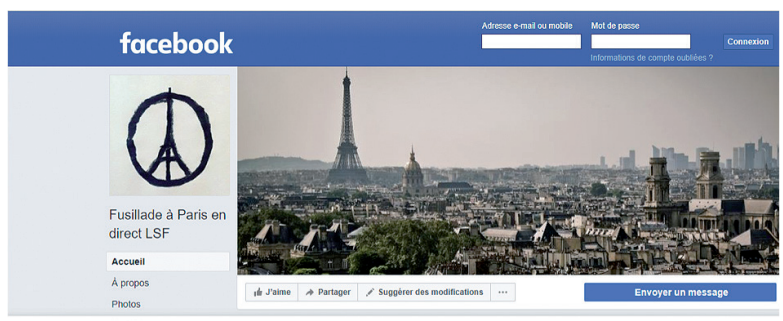

Voir plus de contenu de Fusillade à Paris en direct LSF sur Facebook

$$
\text { Connexion }
$$

$$
\text { Creer un compte }
$$

Figure 1. Page Facebook intitulée « Fusillade à Paris en direct LSF ». prètes LSF (entendants), qui interprètent en simultanée un discours oral, et de traducteurs LSF-vidéo (sourds), qui traduisent à partir d'un support écrit.

Une des particularités de la traduction LSF dans ce contexte d'attentat sur le territoire français réside dans l'engagement des traducteurs sourds français diplômés qui ont accepté de travailler bénévolement. Ils ont mis leurs compétences au service de leur propre communauté sourde, venant ainsi pallier le manquement du gouvernement dans une situation extrême. Au regard de cette situation, nous pouvons nous poser les questions suivantes: traduire en LSF est-il un acte militant?

2. Voir l'enquête Insee «Handicaps-Incapacités-Dépendance», 1998-1999. À notre connaissance, il n'existe aucune étude plus récente à ce sujet.

3. Le rapport Gillot (1998) estimait que $80 \%$ des personnes sourdes étaient en situation d'illettrisme. À notre connaissance, il n'existe aucune étude plus récente, mais ce chiffre est certainement à nuancer aujourd'hui, en raison des avancées didactiques du français écrit pour les personnes sourdes.

4. WwW.facebook.com/Fusillade-à-Paris-en-direct-LSF-315769835260290; suivie par 8779 internautes (consulté le 13 mai 2019). 
Sans être traducteurs en zone de conflit, les traducteurs sourds sont-ils en lutte pour leur reconnaissance? Si oui, quel est leur combat? Enfin, quid de la déontologie propre aux métiers de la traduction quand engagement personnel et projet professionnel s'entremêlent? Pour répondre à ces questions, nous avons parcouru le contenu de la quinzaine de vidéos postées sur la page Facebook susmentionnée, et avons également rencontré courant 2016 des traducteurs sourds, dont deux impliqués dans cette initiative, lors d'entretiens semi-dirigés.

Après un bref historique de la naissance du métier de traducteur sourd, nous présenterons le contexte d'ancrage militant de la communauté sourde en France. Enfin, nous regarderons avec plus d'attention le rendu final de ces traductions-vidéo, sur le fond et sur la forme.

\section{La naissance du métier de traducteur sourd}

En France, la professionnalisation du métier de traducteur sourd est concomitante avec la création de l'entreprise Websourd, aujourd'hui fermée à la suite d'une liquidation judiciaire. Ce premier site internet entièrement bilingue (français écrit et LSF-vidéo) voit le jour en 2004 , avec pour finalité «[...] de contribuer à la citoyenneté des sourds en promouvant la langue des signes ${ }^{5}$ ». II traduisait en LSF-vidéo les brèves journalistiques de l'Agence France-Presse (AFP), entre autres. Au lieu de faire appel à des interprètes, les fondateurs de Websourd, dont des interprètes LSF de profession, ont souhaité solliciter des personnes traduisant vers leur langue première, voire maternelle ${ }^{6}$ et ainsi faire appel à des personnes sourdes. Cependant, à cette époque, aucune personne sourde n'était formée à la traduction. Websourd et le Centre de traduction, interprétation et médiation linguistique (Cetim) de Toulouse créent alors une formation de traducteur en alternance à destination des personnes sourdes.

Jusqu'en 2015, tous les traducteurs sourds formés par le Cetim exerçaient ou avaient exercé à Websourd, partageant une même façon de travailler. De plus, les sourds français avaient un point

5. Voir Gache, 2005; Pelhate, 2009; Fontvieille, 2010.

6. $95 \%$ des personnes sourdes naissent de parents entendants ne pratiquant pas nécessairement la LSF. Ainsi, à défaut de parler de langue maternelle pour les personnes sourdes, on emploie les expressions «langue première» ou «langue d'usage principale». 
d'accès sûr et régulier à l'information en LSF. À la fermeture du site Websourd, les personnes sourdes ne disposaient plus que d'un seul média: la télévision. Cela a laissé un vide, comblé en partie par l'interprétation en LSF des journaux télévisés.

\section{Une communauté sourde issue du militantisme}

Les traducteurs sourds font partie de la communauté sourde - laquelle s'est notamment rendue visible par son militantisme au service de la reconnaissance de sa langue, la LSF - et défendent leur citoyenneté à part entière: "Le développement de la langue des signes est un besoin pour les Sourds, une richesse pour la société7.» À la fin des années 1970, les sourds français, influencés par la situation prospère des sourds américains et leur Deaf Power ${ }^{8}$, ainsi que par le développement des recherches en sciences sociales et humaines sur la langue des signes, militent pour une pleine reconnaissance de leur langue, la LSF9. On parle communément de militantisme sourd, qui culmine avec la reconnaissance de la LSF en tant que langue officielle en France, en février 2005. Nous sommes loin de l'image du militantisme violent, armes à la main. II s'agit de "[...] protester, soulager une souffrance, faire valoir un droit, se rendre visible ou faire entendre sa voix dans un espace public ${ }^{10} \gg$. Le militantisme sourd est un militantisme linguistique (reconnaissance de la LSF), humain (acceptation des sourds tels qu'ils sont) et social (pleine intégration des sourds dans la société), soutenu par un engagement actif (création d'associations de valorisation de la LSF, création d'une théâtralité en LSF, etc.) autour d'une cause commune: le développement identitaire des sourds à travers leur langue.

Ce militantisme sourd a été mené de front avec des entendants bilingues (français et LSF), qui traduisaient bénévolement les différents échanges entre sourds et entendants. Ils ont appris le métier d'interprète sur le tas et ont ressenti le besoin de se former, d'harmoniser leurs pratiques, et de protéger aussi bien les usagers que leur profession naissante, en se conformant

7. Voir Virole, 2000.

8. Le Deaf Power est un mouvement sourd revendicatif, né dans les années 1960, qui se mobilise pour la culture, la langue et les droits des personnes sourdes aux États-Unis.

9. Pour un bref historique de la situation, voir Wirth, 2009.

10. Voir lon, Franguiadakis et al., 2005. 
à un code de déontologie propre". Si les premiers interprètes pouvaient être assimilés à la communauté sourde du fait de leur implication bénévole lors des différentes actions militantes de cette communauté, ce n'est plus le cas aujourd'hui: l'interprète n'est plus un interprète pour les sourds, mais un interprète LSF'2. Ce positionnement est linguistique; il est propre au métier.

Dans cette même optique, le métier de traducteur sourd s'inscrit dans un ancrage linguistique. Cependant, l'appellation "traducteur sourd» peut porter à confusion. Non seulement le traducteur est sourd et agit donc au bénéfice de l'histoire militante de sa communauté (il est même peut-être militant à titre privé?), mais il ne traduit qu'à destination d'un seul public cible: les sourds, sa propre communauté. La distance interprète (entendant)/communauté sourde et le fait d'interpréter à destination de deux publics cibles différents (sourds et entendants) n'existent pas en tant que tels chez les traducteurs sourds. Ceux-ci ne traduisent que très rarement vers le français écrit; la majorité de leur pratique traduisante se déroule vers la LSF-vidéo. De plus, si l'ancienne appellation d'«interprète pour sourds» mettait l'emphase sur une notion d'assistanat de la communauté sourde par les interprètes, celle de "traducteur sourd» se focalise sur l'aspect culturel du traducteur ${ }^{13}$. Ce dernier s'exprime en LSF avec aisance et il est à même de traduire en tenant compte des aspects sociolinguistiques de son public cible, qui est aussi sa communauté.

L'identité des sourds est intrinsèquement liée à leur langue naturelle, la LSF. Au cours de nos entretiens avec différents traducteurs sourds, seul l'un d'eux s'est revendiqué comme traducteur F/LSF-vidéo et non traducteur sourd, jugeant cette dernière appellation trop stigmatisante, et allant jusqu'à ironiser: "On ne parle pas d'interprète entendant non plus!»

\section{Les choix du traducteur sourd}

\section{Le contenu}

En parcourant le contenu de la page Facebook "Fusillade à Paris en direct LSF», on remarque non seulement que les

11. Voir Bernard, Encrevé et al., 2007.

12. Voir Jeggli, 2003.

13. Concernant la culture sourde, voir Mottez, 2006. 
allocutions du président sont interprétées, mais également que d'autres textes à thématique géopolitique sont traduits. Ces textes viennent éclairer la conjoncture d'attentat. En effet, si le contexte géopolitique de ces attaques terroristes n'est pas nécessairement connu de tous les entendants, il leur est du moins plus facilement accessible. L'équipe mise en place, consciente des probables lacunes de sa communauté (sourde), ne se contente pas de traduire les articles de journaux actuels, mais fait le choix de traduire également d'autres textes pertinents qui permettent de contextualiser la situation. Ce choix de traduire des textes qui ne sont pas directement liés aux attentats émane des journalistes, et non des traducteurs.

\section{La mise en forme}

Chaque vidéo commence par le titre du texte source, l'auteur du texte, et le nom du traducteur sourd; c'est la première image qui apparaît à l'écran en début de traduction vidéo (voir figure 2).

Publé par Fusillade à Paris en direct LSF

Le point sur la Syrie

Un texte écrit par Elsa Ponchon
traduit par Vivien Fontvieille

Figure 2. Exemple d'image introduisant chaque traduction vidéo.
La distinction entre journaliste et traducteur est claire: le journaliste écrit le texte, le traducteur le traduit. II est systématiquement mentionné que le récepteur de l'information se trouve face à une traduction ou à une production directement réalisée en LSF (par les journalistes sourds). Là encore, les journalistes sont responsables de la mise en forme de la vidéo.

Cependant, compte tenu du caractère récent de la professionnalisation du métier de traducteur en LSF-vidéo, la confusion peut demeurer pour certains (sourds et entendants) qui, voyant une personne sourde signer à l'écran, pensent qu'elle s'exprime en production spontanée, en son nom propre. En effet, la traduction en LSF-vidéo ne laisse pas de place à l'anonymat du traducteur. L'incarnation physique de la traduction, du fait de la modalité visuo-gestuelle de la LSF, permet d'identifier le 
traducteur immédiatement ${ }^{14}$. Ainsi, les remarques, voire réclamations de quelques internautes sur le contenu de la vidéo étaient dirigées vers le traducteur, et non vers le journaliste - auteur non visible du texte source. Les traducteurs ont dû, parfois, intervenir eux-mêmes pour préciser que toute erreur de données devait être rapportée directement au journaliste. Le traducteur sourd Vivien Fontvieille a ainsi réagi à un commentaire le visant, en date du 23 novembre 2015:

[...] Petit rappel: je l'ai juste traduit en LSF pour que les informations de cet article vous soient accessibles. En effet, je ne me suis en aucun cas trompé car ce n'est pas moi qui l'ai écrit. Sachez que je suis soumis à la règle de déontologie de mon métier, je reste donc fidèle en LSF au texte français. Et attention à ne pas confondre deux métiers: traducteur et journaliste. [...]

Malgré cette justification du traducteur sourd, les critiques ont persisté. Un internaute sourd a renchéri en répondant: «[...] Mais ne sois pas un mouton, à traduire sans vérifier!» Cette réaction peut être révélatrice de la méconnaissance du métier de traducteur sourd, même au sein de la communauté sourde - voire de la méconnaissance du métier de traducteur tout court. De même, les professions liées à la traduction/interprétation sont généralement associées à un métier d'entendant. Les formations pour traducteurs LSF (sourds) sont beaucoup plus récentes que celles des interprètes LSF (entendants) : 2005 contre fin des années 1980.

\section{La dactylologie}

Du point de vue de l'acte de traduction lui-même, le métier de traducteur sourd se rapproche de celui de traducteur en langue vocale écrite dans la mesure où il s'agit avant tout de transmettre du sens. Une des spécificités de la traduction finale réside dans cette présence persistante de la langue source, soit le français écrit. En effet, si les noms propres sont dactylologiés (épelés en LSF), ils sont aussi affichés en français et écrits en haut à gauche de l'écran, avant ou après la traduction en LSF. Le nom est dactylologié en LSF, puis un temps de pause est observé pour

14. Voir Encrevé, 2019. 
laisser le temps de lire le nom écrit. Enfin, la traduction reprend. Ce choix émane des traducteurs, sourds eux-mêmes, et donc conscients des difficultés de compréhension de la dactylologie par le lecteur sourd, en raison de la rapidité d'exécution et/ou du paramètre écran qui gêne la réception visuelle.

\section{L'ajout d'images}

Une autre spécificité apparaît dans le résultat final de la traduction. Il s'agit de l'ajout de l'image du drapeau d'un pays ou bien du visage d'une personnalité publique lorsqu'un nom propre est traduit (voir figure 3). Ce choix émane des traducteurs sourds, soucieux d'une réception optimale par le public cible, et conscients de la construction visuelle des sourds et des poten-

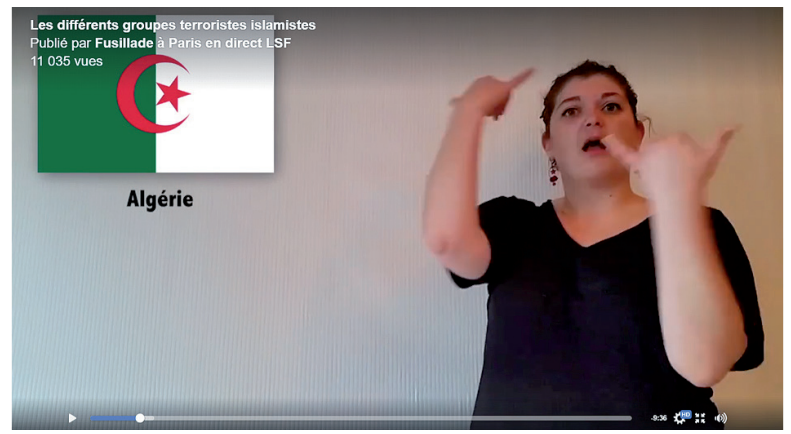

Figure 3. Exemple d'une traductrice sourde signant " Algérie " pendant que le drapeau et le nom écrit du pays apparaissent à l'écran. tielles lacunes en termes d'écrit; ils n'hésitent donc pas à ajouter des éléments extralinguistiques à la traduction, qui peuvent s'apparenter à des notes de bas de page. Les personnes sourdes peuvent ainsi faire le lien entre le signe en LSF, le mot français (auquel il a sûrement déjà été confronté précédemment), et le drapeau ou le visage d'une personnalité publique comme support visuel d'aide à la compréhension, puisqu'il rappelle peut-être un élément déjà vu ou connu.

\section{Conclusion}

Ce partenariat journaliste-traducteur semble bien rodé; il est ancré dans l'histoire du métier de traducteur sourd depuis l'époque de Websourd. Traducteurs et journalistes collaboraient déjà régulièrement. Cependant, si la profession de traducteur sourd semble organisée et claire quant à son positionnement dans le domaine de la traduction et non du journalisme, elle reste tributaire d'un manque de connaissances et de reconnaissance, 
que ce soit par les personnes sourdes et entendantes, ou de façon plus générale, par les autorités françaises.

L'appellation "traducteur sourd» peut laisser penser que le traducteur n'intervient pas sur le côté linguistique, mais sur le versant de l'assistanat. Or l'appellation «traducteur sourd» doit être considérée du point de vue sociolinguistique; le sourd fait partie de la communauté sourde et en connaît les codes, dont la langue des signes. Lorsqu'il maîtrise la LSF (langue première) et le français écrit (langue seconde) et qu'il est formé aux techniques de la traduction, il peut alors devenir traducteur en respectant la déontologie du métier.

Les traducteurs engagés, exerçant bénévolement, ne sont pas pour autant moins professionnels. Ils restent fidèles à leur métier et à leur rôle de professionnels linguistiques et culturels. Ils ne sont pas traducteurs de guerre, mais pourraient être considérés comme tels, malgré tout et malgré eux. Ils sont en effet en guerre contre l'illettrisme: la traduction en langue des signes reste un outil d'accessibilité indispensable pour les personnes sourdes, et non un choix linguistique entre le texte français et la LSF; la majorité des sourds n'est pas (encore?) bilingue. Et en guerre contre l'exclusion sociale des personnes sourdes; car les retombées de la loi de 2005 tardant à se faire ressentir, traduire revient à essayer de diminuer cette exclusion.

Les traducteurs sourds sont engagés et professionnels, mais par la force des choses bénévoles, car oubliés d'une partie de la société. À l'instar du métier d'interprète LSF qui s'est professionnalisé il y a une quarantaine d'années et commence seulement à bénéficier d'une plus grande visibilité et d'une meilleure rémunération, le métier de traducteur sourd LSF n'en est qu'à ses débuts et doit encore faire ses «preuves» en passant par le bénévolat, avant d'entrer pleinement dans le paysage sociolinguistique et professionnel de la France.

aurelia.ngg@gmail.com 
Aurélia Nana Gassa Gonga est interprète français-LSF diplômée de I'Université Paris 8 depuis 2012. Alliant pratique de I'interprétation et recherche sur l'interprétation, c'est au travers de la linguistique des langues des signes qu'elle s'intéresse aux stratégies d'interprétation des traducteurs ou interprètes en langue des signes, qu'ils soient sourds ou entendants, en France ou à l'étranger. Actuellement doctorante en linguistique des langues des signes à I'université de Radboud, aux PaysBas, elle s'intéresse particulièrement aux stratégies d'interprétation adoptées par les interprètes dont une des langues de travail est les «signes internationaux». Membre du conseil d'administration de I'Association française des interprètes et traducteurs en langue des signes (AFILS), elle agit en faveur d'une meilleure reconnaissance du métier en France et à l'international.

\section{SOURCES CITÉES}

BERNARD Alexandre, ENCREVÉ Florence et al., L'Interprétation en langue des signes, Paris, Puf, 2007.

ENCREVÉ Florence, «L'incarnation des interprètes et des traducteurs LSF/français lorsqu'ils œuvrent vers la langue des signes", in HIBBS Solange, SERBAN Adriana, VINCENT-ARNAUD Nathalie (dir.), Corps et traduction, Corps en traduction, Limoges (France), Lambert-Lucas, 2019, p. 79-92.

FONTVIEILLE Vivien, Métier traducteur LSF: un nouveau métier, quels travaux, quelles techniques?, mémoire de master Traduction, Interprétation, et Médiation linguistique, Université Toulouse 2, non publié, 2010.

GACHE Patrick, La traduction français-écrit/langue des signes vidéo, mémoire de master Interprétation en langue des signes, Université Lille 3, non publié, 2005.

GILLOT Dominique, Rapport «Le droit de sourds, 115 propositions. Rapport parlementaire au Premier ministre», Paris, 1998.

ION Jacques, FRANGUIADAKIS Spyros et al., Militer aujourd'hui, Paris, Autrement, 2005.

JEGGLI Francis, «L'interprétation Français/LSF à l’université», in Langue française, 137, 2003, p. 114-123.

MOTTEZ Bernard, Les Sourds existent-ils?, Paris, L'Harmattan, 2006.

PELHATE Julia, Traducteur LSF: un nouveau métier, quel cadre, quelles limites? La traduction hors cadre: au théâtre, mémoire de master Traduction, Interprétation et Médiation linguistique, Université Toulouse 2, non publié, 2009.

QUIPOURT Christine et GACHE Patrick, «Interpréter en langue des signes: un acte militant?", in Langue française, 137, 2003, p. 105-113.

VIROLE Benoît, Psychologie de la surdité, Bruxelles, De Boeck, 2000.

WIRTH Françoise, «Interprètes en langue des signes: autour de l'intervention de MarieThérèse L'Huillier à la Journée mondiale de la Traduction 2008", in Traduire, 221, 2009, p. 33-38. 